\title{
Complex System Fault Diagnosis Method Based on CFFPN
}

\author{
Yan $\mathrm{Su}^{1,} \mathrm{a}^{*}$, Yiqing Ling ${ }^{2, \mathrm{~b}}$ and Hui Wang ${ }^{3, \mathrm{c}}$ \\ ${ }^{1,2}$ Nanjing University of Aeronautics and Astronautics, Nanjing, Jiangsu, 210016 \\ asuyannj@nuaa.edu.cn, blingyiqin@nuaa.edu.cn, cwanghuinuaa@nuaa.edu.cn
}

Keywords: Colored fuzzy fault petri net ( CFFPN ) ; Fault diagnosis; Modeling; Complex system; Pneumatic system

\begin{abstract}
Considering the complexity of system structure and fault mechanism of complex system, a fault diagnosis method based on extended color fuzzy fault Petri net model is proposed (CFFPN) for civil aircraft. In order to increase the fault diagnosis efficiency, the CFFPN is modeled based on fuzzy mathematics and fault Petri net method through adding token, library and staining rules to describe the fault propagation. Fuzzy generated rules combined with matrix theory are utilized for fault diagnosis reasoning. CFFPN based reasoning algorithm is given. The validity of the proposed method is verified in one type aircraft pneumatic system.
\end{abstract}

\section{Introduction}

With the rapid development of mechanical and electronic technologies, electro-mechanical systems have become more complex. At present, a variety of fault diagnosis models have been established to describe the failure process of complex systems, such as Hidden Markov Model(HMM)[1], Gray Model(GM) [2], Bayesian Model [3], Back Propagation(BP) [4],Signed Directed Graph(SDG)[5], Multi-Signal Flow Graph(MSFG)[6,7], Petri-based Fault Diagnosis Model[8] and so on. Fault diagnosis and analysis method is divided into 3 development stages, i.e. qualitative analysis, quantitative calculation, and qualitative and quantitative method based on fuzzy mathematics. For that fuzzy mathematical method can well reflect the existing fuzzy phenomena and Petri net is one rich system description and system behavior analysis means with strict mathematical expression and intuitive graphical expression, the method of combining fuzzy mathematics with Petri Net has been widely used in recent years $[9,10,11]$. But these methods can not well describe the fault propagation characteristics. Parameters of complex system usually are various and mutual influence. Complex system structure and fault propagation state expression are very complex. System faults usually appear the features of ambiguity, uncertainty, stochastic, dynamic and non quantitative.

In order to make the fault information expression more complete and also well present the fault propagation path, a colored fuzzy fault petri net (CFFPN) modeling method is proposed. A matrix reasoning algorithm based on CFFPN is established. It is applied in one type aircraft pneumatic system. The proposed method can improve the efficiency and effectiveness of the diagnosis and troubleshooting.

\section{CFFPN Model}

CFFPN mathematical model is described by twelve elements:

$$
S_{C F F P N}=(P, T, \boldsymbol{I}, \boldsymbol{O}, K, C, \boldsymbol{M}, \boldsymbol{\Omega}, \boldsymbol{\alpha}, f, \boldsymbol{H}, \boldsymbol{U})
$$

Wherein, $P=\left\{p_{1}, p_{2}, \cdots, p_{n}\right\}(n>0)$ is the set of fault events and $p_{i}$ is library, $T=\left\{t_{1}, t_{2}, \cdots, t_{m}\right\}(m>0)$ the set of state change nodes and $t_{j}$ state change node, $I=P \times T$ describe the mapping from library to change node and $I=\left\{\delta_{i j}\right\}$ and $\delta_{i j}=1$ or $0, \boldsymbol{O}=T \times P$ describe the 
mapping from change node to library; $\boldsymbol{O}=\left\{\gamma_{i j}\right\}, \gamma_{i j}=1$ or $0, K=\left\{k_{1}, k_{2}, \cdots, k_{n}\right\}$ is set of Token which represents confidence level of fault events and $C$ the set of color, $\boldsymbol{M}=\left(m_{1}, m_{2}, \cdots, m_{n}\right)^{\mathrm{T}}$, is the set of number and color of Token for each library $p_{i}, \boldsymbol{\Omega}=\left(\omega_{1}, \omega_{2}, \cdots, \omega_{n}\right)^{\mathrm{T}}$ express the effect to change rules of $t$ by input library $p, \alpha=\left(\alpha_{1}, \alpha_{2}, \cdots, \alpha_{n}\right)^{\mathrm{T}}$ is confidence vector for fault events and $\boldsymbol{\alpha}_{i}=[0,1], f=\left\{f_{1}, f_{2}, \cdots, f_{n}\right\}$ is the set of fuzzy failure rate of the base event, $\boldsymbol{H}=\left(\lambda_{1}, \lambda_{2}, \cdots, \lambda_{m}\right)^{\mathrm{T}}$ is the ignition threshold of change rules of each, $\boldsymbol{U}=\operatorname{diag}\left(\mu_{1}, \mu_{2}, \cdots, \mu_{m}\right)$ is confidence matrix of change rule and $\mu_{j}$ the threshold of $t_{j}$.

CFFPN graph model can be established based on above mathematics model. Firstly, basic Petri net model is established according to the logical relationship of fault events which are got from fuzzy production rules through system structure analysis. Secondly, colored Petri net model is built in accordance with the token staining rules and library stain rules. Finally, the change igniting rules which describe the system state are defined. For fuzzy intelligent fault diagnosis system, fault events confidence range among [0 1], the number of token represent fault propagation path and fault severities and the color of Token confidence levels of fault event. The token staining rules see Table 1 and the library P stain rules are as following.

The library $\mathrm{P}$ staining rules:

If $P \times T \rightarrow N=1, T \times P \rightarrow N=0$, then the library $\mathrm{P}$ is the root cause of failure or fault symptom, and its state is described in signature (O) in graph model.

If $P \times T \rightarrow N=1, T \times P \rightarrow N=1$, then the library $\mathrm{P}$ is the process cause of failure, and its state is described in signature " $\bigcirc$ " in graph model.

If $P \times T \rightarrow N=0, T \times P \rightarrow N=1$, then the library $\mathrm{P}$ is the final failure state or fault state, and its state is described in signature in graph model.

When $p_{i}$ is the input of $t_{j}$, there is a directed connection line from $p_{i}$ to $t_{j}$. When $p_{i}$ is the output of $t_{j}$, there is a directed connection line from $t_{j}$ to $p_{i} . \forall t_{j} \in T$, if $\forall p_{k} \in I\left(t_{j}\right), \sum_{k=1}^{n} \alpha\left(p_{k}\right) \cdot \omega_{k} \cdot \mu\left(t_{j}\right)>\lambda\left(t_{j}\right), j=1,2, \cdots, m$, and $\boldsymbol{M}\left(p_{k}\right) \geq 1, \quad k=1,2, \cdots, n$, then $t_{j}$ is potential enable change node. If $\boldsymbol{M}\left(p_{k}\right) \geq 1$, then $t_{j}$ can trigger ignition and will produce new confidence level. No ignition changes described in black signature $\mathbf{I}$ and ignition changes in red signature I among CFFPN graph model. Fig. 1 is one simple CFFPN graph model.

Table 1. Color rule of Token

\begin{tabular}{|c|c|c|c|c|}
\hline Confidence & Threshold & $\begin{array}{c}\text { Staining } \\
\text { Mark }\end{array}$ & Color & Meaning \\
\hline $1^{\text {th }}$ level & $0.95 \sim 1.00$ & $\star$ & Red & Very true \\
\hline $2^{\text {th }}$ level & $0.90 \sim 0.94$ & & Orange & True \\
\hline $3^{\text {th }}$ level & $0.85 \sim 0.89$ & & Yellow & General true \\
\hline $4^{\text {th }}$ level & $0.70 \sim 0.84$ & $\star$ & Gr a y & Basic true \\
\hline $5^{\text {th }}$ level & $0.50 \sim 0.69$ & $\bullet$ & Blue & Not too true \\
\hline $6^{\text {th }}$ level & $0.00 \sim 0.50$ & $\nabla$ & Purple & Untrue \\
\hline
\end{tabular}




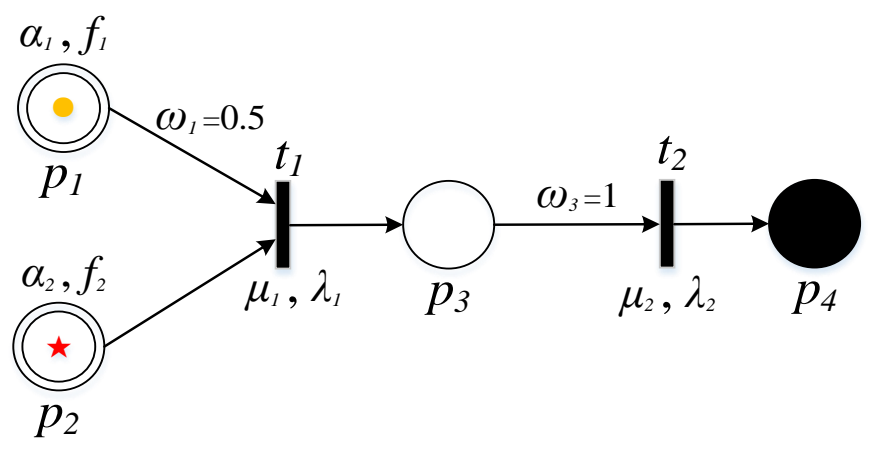

Figure 1. CFFPN graph model

\section{CFFPN Based Fault Diagnosis}

In order to provide the necessary condition for fault evaluation and diagnosis, $\mathrm{MYCIN}^{[11]}$ is utilized to get the confidence value of all the faults in the library. The confidence level of $p_{i}$ is calculated in Eq. 2. The calculation is ended till $\boldsymbol{\alpha}_{k+1}=\boldsymbol{\alpha}_{k}$.

$$
\boldsymbol{\alpha}_{k+1}=\boldsymbol{\alpha}_{k} \oplus\left[(\boldsymbol{O} \cdot \boldsymbol{U}) \otimes\left(\overline{\boldsymbol{I}^{\mathrm{T}} \otimes \overline{\boldsymbol{\alpha}_{k}}}\right)\right]
$$

CFFPN based diagnosis algorithm is as following.

$$
\begin{aligned}
& U\left(t_{j}\right)=\frac{1}{\left.1+e^{-b\left[\sum_{k=1}^{n} \alpha\left(p_{k}\right) \cdot \omega_{k} \cdot \mu\left(t_{j}\right)-\lambda\left(t_{j}\right)\right.}\right]}, j=1,2, \cdots, m \\
& \left\{\begin{array}{rlrl}
\boldsymbol{I}_{k} & =\boldsymbol{I}^{\mathrm{T}} * \boldsymbol{M}_{k-1} & & k=1 \\
\boldsymbol{I}_{k}=\boldsymbol{I}^{\mathrm{T}} *\left(\boldsymbol{M}_{k-1}-\boldsymbol{M}_{k-2}\right) & & k=2,3, \ldots
\end{array}\right. \\
& \boldsymbol{U}_{k}=\boldsymbol{U} \wedge\left(\left(\boldsymbol{I}_{k} \wedge \boldsymbol{l}_{n \times m}\right)^{\mathrm{T}} \circ \boldsymbol{\Omega}\right) \quad k=1,2,3, \ldots \\
& \boldsymbol{M}_{k}=\boldsymbol{M}_{k-1}+\left(\boldsymbol{O}\left(\left(\boldsymbol{I}_{k}{ }^{\mathrm{T}} \square \boldsymbol{M}_{k-1}\right)^{\mathrm{T}} * \boldsymbol{U}_{k}\right)\right), k=1,2,3, \cdots \\
& \boldsymbol{U}(t)=\left(U\left(t_{1}\right), U\left(t_{2}\right), \ldots, U\left(t_{m}\right)\right)^{\mathrm{T}}
\end{aligned}
$$

\section{Application}

In order to verify the validity, the proposed method is applied in one type aircraft pneumatic system and a CFFPN model of aircraft pneumatic system is established. Aircraft pneumatic system is a complex system, and inter-influence of its fault is very complex. The established CFFPN model is very big. Here just a part of the CFFPN model is selected to verify the validity. Fig.2 is the CFFPN model of one subsystem of aircraft pneumatic system, table 3 present relevant fault events. It can clearly express the fault propagation process.

According to the expert knowledge base and historical data, set the initial value as follows

$$
\begin{aligned}
& \boldsymbol{a}_{0}=(0.95,0.9,0.7,0.86 .0 .95,0.82,0.8,0,0,0,0,0,0)^{\mathrm{T}}, \boldsymbol{\Omega}=(1,1,1,1,1,1,1,1,1,1,1,1,0)^{\mathrm{T}}, \\
& \boldsymbol{U}=\operatorname{diag}(0.9,0.8,0.75,0,8,0.85,0.7,0.85,0.9,0.95,0.9,0.8,0.9) .
\end{aligned}
$$

When the APU bleed air system work, four kinds of fault symptoms are predicted through testing, i.e. APU bleed air over temperature, APULCV open degree small, APULCV off fail and HPGCV open fail. According Token staining rule, Fig. 2 is renewed and the original library is produced, shown in Fig. 3. Set original state $\boldsymbol{M}_{0}=(0,1,0,1,1,1,0,0,0,0,0,0,0)^{\mathrm{T}}$. Final token state is obtained through calculation by Eq.3, Eq.4, Eq.5, see Fig. 3. Relevant fault severity, propagation path and confidence level see table 2 . It can clearly see that $p_{8}, p_{9}, p_{10}, p_{11}, p_{12}, p_{13}$ are the possible 
faults produced by fault symptoms. Maintenance engineers can directly inspect these components and thus shorten the time of troubleshooting.

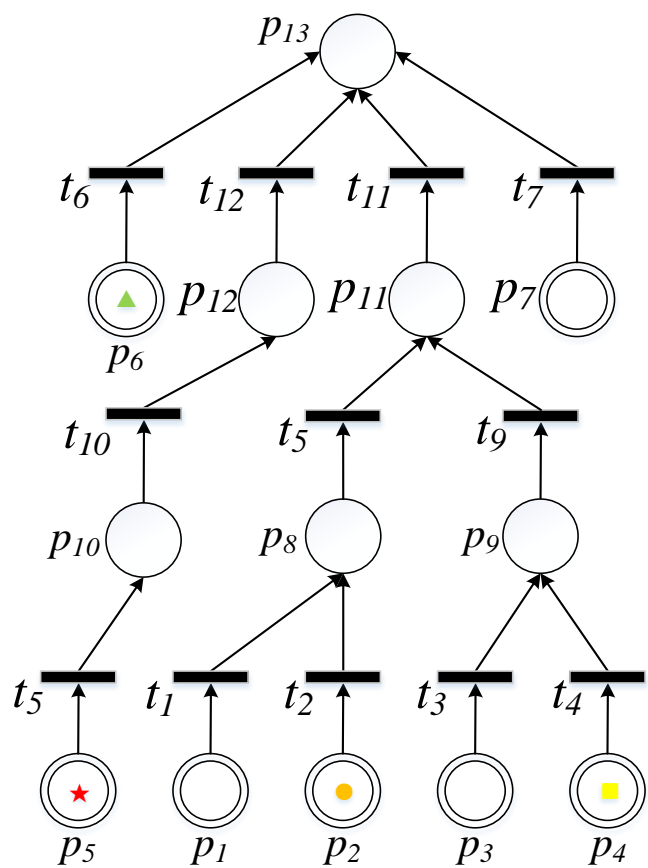

Figure 2. Part CFFPN model Of aircraft pneumatic system

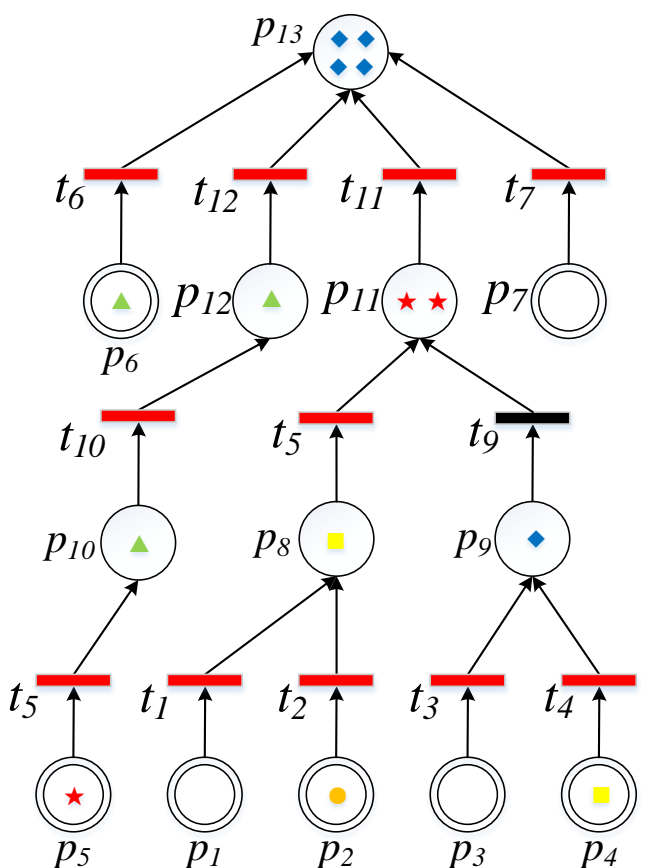

Figure 3. Token final states

Table 2. Fault propagation state information

\begin{tabular}{|c|c|c|c|c|c|c|}
\hline Library & $p_{8}$ & $p_{9}$ & $p_{10}$ & $p_{11}$ & $p_{12}$ & $p_{13}$ \\
\hline Severity & 1 & 1 & 1 & 2 & 1 & 4 \\
\hline $\begin{array}{c}\text { Propagation } \\
\text { path }\end{array}$ & $p_{1} \rightarrow p_{8}$ & $p_{4} \rightarrow p_{9}$ & $p_{5} \rightarrow$ & $p_{1} \rightarrow p_{8} \rightarrow p_{11}$ & $p_{5} \rightarrow p_{10}$ \\
$p_{10} \rightarrow p_{9} \rightarrow p_{11}$ & $\begin{array}{c}p_{12} \rightarrow p_{8} \rightarrow p_{11} \rightarrow p_{13} \\
p_{4} \rightarrow p_{9} \rightarrow p_{11} \rightarrow p_{13} \\
p_{5} \rightarrow p_{10} \rightarrow p_{12} \rightarrow p_{13} \\
p_{6} \rightarrow p_{13}\end{array}$ \\
\hline $\begin{array}{c}\text { Confidence } \\
\text { level }\end{array}$ & $3^{\text {th }}$ level & $5^{\text {th }}$ level & $4^{\text {th }}$ level & $1^{\text {th }}$ level & $4^{\text {th }}$ level & $5^{\text {th }}$ level \\
\hline
\end{tabular}

\section{Conclusions}

In this paper, the proposed CFFPN model established by introducing staining rules to fuzzy generation rules can very well express the fault propagation characteristics. Transition ignition matrix reasoning method based on CFFPN model can make the maintenance engineer quickly determine the fault, and effectively improve diagnoses and troubleshooting efficiency.

\section{Acknowledgements}

This research was financially supported by the National Natural Science Foundation (U1233114).

\section{References}

[1] L. Atlas, M. Ostendorf, G.D. Bernard. Hidden Markov models for monitoring machining tool-wear [J]. IEEE Acoustics, Speech, and Signal Processing, 2000,6(6):3887-3890. 
[2] Deng JL. Basic method for gray system [M]. Wuhan: Huazhong Science and Technology Press, 2005.

[3] P. Weber, D. Theilliol, C. Aubrun. A. Evsukoff. Increasing effectiveness of model-based fault diagnosis: A Dynamic Bayesian Network design for decision making [J]. Fault Detection Supervision \& Safety of Technical Processes, 2006, 6(1):90-95

[4] He Yigang, Li Yun, Liu Meirong. BP neural network approach to module fault diagnosis for large analog circuit [J]. In: Proceeding of TENCON 2004. IEEE Region 10 Con-ference, 2004, 4: 395-398.

[5] Yan Su. Semiquantitative SDG Graphical Modeling for Complex System Fault Diagnosis [J]. Applied Mechanics and Materials, 2012, 152-154:1601-1606.

[6] Somnath Deb, Krishna R Pattipati, Vijay Raghavan, et al. Multi- signal flow graphs: a novel approach for system testability analysis and fault diagnosis [J]. Aerospace and Electronic Systems Magazine, 1995,10, ( 5) : 14-25.

[7] SU Yan, LIU Pengpeng. Multi-hierarchical Functional Directed Graph Modeling Method for Aircraft System Fault Diagnosis [J]. Applied Mechanics and Materials, 2014, 541-542:1467-1472.

[8] Lo K L, Ng H S, Trecat J. Power systems fault diagnosis using Petri nets [J]. IEE Proceedings_-Generations, Transmissions and Distributions, 1997,144(3):231-236.

[9] Adamyan A, He D. System failure analysis through counters of Petri Net models [J]. Quality and Reliability Engineering International, 2004, 20(4):317-335.

[10]Cheng Xuezhen, Wang Cheng, Yu Yongjin, Yi Lifeng, Chen Qiang. Three-phase asynchronous motor fault diagnosis method based on Fuzzy fault Petri net [J]. Transactions of China Electro technical Society, 2015, 17:132-139.

[11]ZENG Qing-feng, HE Zheng-you, YANG Jian-wei. Fault diagnosis model of electric power systems based on coloured Petri net. [J]. Power System Protection and Control, 2010, 38(14):5-11. 